\title{
Body Fat Percentage Extracted from 3-D Scans for Sports \& Medical Science
}

\author{
Jochen BALZULAT ${ }^{\mathrm{a}}{ }^{*}$, Ulrich BOTZENHARDT ${ }^{\mathrm{a}}$, Norbert BACHL ${ }^{\mathrm{b}}$, Arnold BACA $^{\mathrm{b}}$, Mario HELLER $^{\mathrm{b}}$ \\ ${ }^{a}$ Human Solutions $\mathrm{GmbH}$, Kaiserslautern, Germany \\ ${ }^{\mathrm{b}}$ Zentrum für Sportwissenschaft und Universitätssport an der Universität Wien, Austria
}

\begin{abstract}
Body scanning technologies are used in many applications, from anthropometric research to garment technology to biomechanics. While capturing a subject's body shape and measurements is often the primary objective, 3-D body scan systems can potentially add more value in some applications by determining additional body characteristics. In sports and medical science those are body mass, body surface, or overall body fat percentage.

To develop algorithms capable of calculating such values, an extensive survey was conducted in which subjects were scanned in various positions to develop a stable and practicable process that considers process-inherent perturbations. Also, additional values were collected and interviews conducted to correlate subjects' diet and activity with body characteristics.

As a result, a new method was developed that allows estimating overall body fat percentage based on 3-D scans. The new method was evaluated against widely used methodologies such as infrared interactance, skin fold measurements, and body impedance.
\end{abstract}

Keywords: 3-D body scanning, body fat percentage, densitometry, body volume, body mass

\section{Introduction}

The relatively new technology of 3-D body scanning offers a wide area of applications. Obviously the benefit of a 3-D scan is not only the visual representation of a human body but also information that can be extracted from such 3-D shapes. While some applications use 3-D scans for visualization e.g. the movie industry, others address the use of 3-D shapes in order to gain additional information on the scanned individual.

One focus of body scanning lies in applications for the garment industry. Scans are commonly used to determine body measurements for garment size tables, made-to-measure items, and fitting solutions for garment logistics or to provide 3-D surface information for virtual-draping simulations. Lately, other disciplines such as sports science, medicine, and biomechanics have shown interest in using 3-D body scanning technologies.

Applications of 3-D body scanners in the latter fields widely vary, although the most common request by users is to determine additional body characteristics on the basis of 3-D scans other than body measurements. Body characteristics like total surface and composition may be used for the design of sporting equipment, biomechanics, sports medicine as well as for promoting health, quantifying performance, talent scouting and many more applications.

\section{Method}

\subsection{Objective}

The target of this project is to establish a process and an approach that allows estimating the overall body fat percentage based on 3-D scans. This new approach shall be compared with existing methods.

The resulting method shall be valid for males and females featuring a wide range of body sizes and body shapes. Also, it is important that the necessary measuring process shall work in common 3-D body scanning set-ups, i.e. it must be user-friendly, stable, practicable, and must consider processinherent perturbations.

\footnotetext{
* jochen.balzulat@human-solutions.com; +49 631 3035600; www.human-solutions.com
} 


\subsection{Approach}

There are various approaches to measure body fat [5]. Some of the most common ones are imaging methods like dual-emission X-ray absorptiometry (DEXA), measuring subcutaneous fat tissues by directly determining the tissue thickness (skin fold method) or indirectly with help of IR light that is emitted on and reflected by the human body (IR interactance). Another set of procedures can be summarized in methods of densitometry. Densitometry refers to methods that estimate body composition from body density [4]. The more information is available to estimate body density, e.g. fraction of total body water, minerals, etc. the more accurate the total body fat percentage. Depending on the number of components used, models are classified as two-component, three-component or even four-component models.

Since the objective is to calculate body fat percentage from a 3-D scan of an individual, obviously models of densitometry are preferred. In principle the body volume can be calculated from a 3-D scan, and together with body mass taken simultaneously, the overall body density may be calculated. Therefore, the two-component body composition model is applied dividing body mass into fat and fatfree masses. Widely used two-component models were published by Siri [7] and Brozek [2].

$$
\begin{aligned}
& \text { Body Fat } \text { Siri }_{\text {net }}=\frac{4,950}{\rho_{\text {net }}}-4,500 \\
& \text { Body Fat } \text { Brozek }_{\text {net }}=\frac{4,570}{\rho_{\text {net }}}-4,142
\end{aligned}
$$

In order to assess this new method it shall be compared with existing ones, namely bioimpedance, skin fold method, and infrared interactance.

Here body fat percentage shall be calculated based on 3-D scans using the above mentioned models. To calculate body density, body mass is divided by the 3-D scan volume. Raw scans consisting of a 3-D point cloud describe the subjects' outer surface, from which the volume may be derived by creating a so-called single-surface watertight triangle mesh (Figure 1).
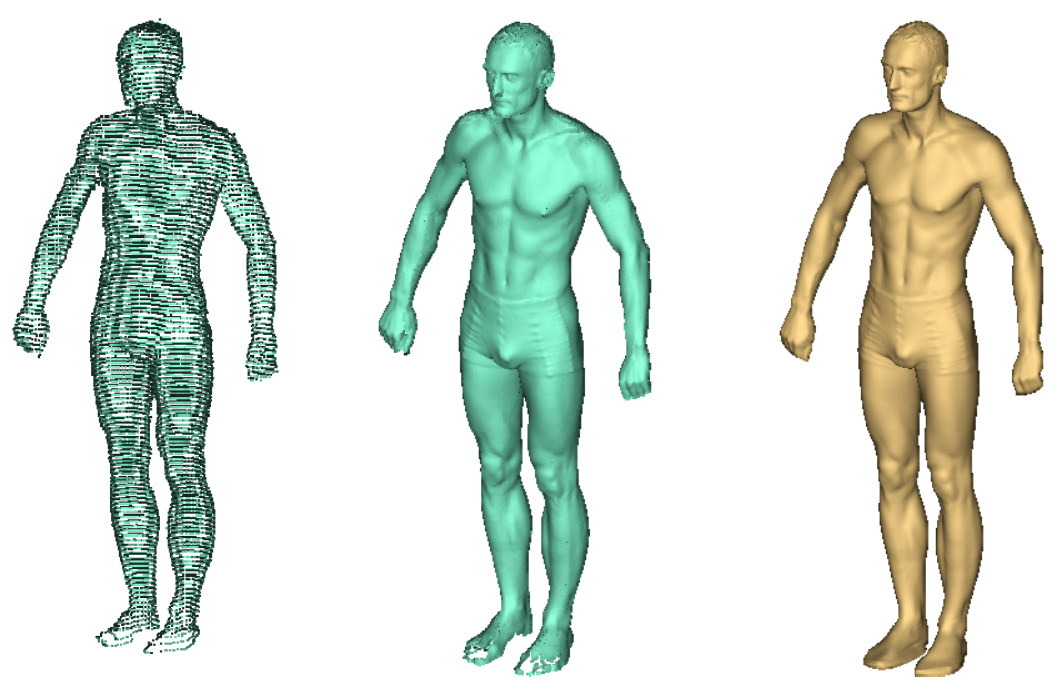

Figure 1: Raw scan, points (le.), raw scan, surfaces (mi.), triangulated watertight mesh (ri.)

Strictly speaking, such meshes include additional pseudo-volume created by body hair, air inclusions in the body, etc. Therefore, the scanning process must be defined in a way such that those volumes are avoided. Some methods such as optimizing the posture in which subjects are scanned in order to correctly model cleavage areas like armpits and crotch, preparing body hair accordingly and wearing proper scan wear can reduce the error. But still, due to air inclusions a 3-D scan gross volume is typically bigger than the true body volume needed and, therefore, must be corrected to calculate body density: 


$$
\begin{aligned}
& V_{\text {net }}=f\left(V_{\text {gross, scan }}\right) \\
& \rho_{\text {net }}=\frac{m}{V_{\text {net }}}
\end{aligned}
$$

\subsection{Set-Up}

The database for this effort are scans, measurements, and personal information of 141 subjects.

Collecting adult male and female subject data in a wide range of body shapes and body dimensions was the focus, and hence data on children were not collected. The minimum number of subjects needed was determined in accordance with ISO 20685 [6], which required 112 subjects. Actually, data of 150 subjects were collected while only 141 data sets finally were used due to quality, completeness checks, etc.

Excluded from the survey were subjects taking certain medications and subjects undergoing extreme diets. But to cover extreme body types bodybuilders for example, were allowed to participate.

To prepare for the experiments subjects were asked not to undergo excessive physical stress on the day of measuring and to follow a controlled ingestion the night before and in the morning of the measuring day. To maintain a natural level of body fluids regular drinking during the measuring day was allowed but no dehydrating fluids such as coffee or alcohol (except for a coffee in the morning) were allowed. Appropriate scan wear, i.e. tight-fitting measuring garments were supplied to the subjects.

The main data collected for each subject were body fat percentage - or equivalent data - with bioimpedance method (1 repetition), infrared interactance (3 repetitions), skin fold method measuring - 4 folds (3 repetitions). 3-D scans in five postures and breathing states were taken: standing relaxed, random breathing state (1 repetition); standing - arms and legs splayed slightly apart, random breathing state (2 repetitions); standing - legs and arm strongly splayed apart, random breathing state. The last was taken in a random breathing state, maximum exhalation, and maximum inhalation (1 repetition for each breathing state).

These multiple scans were taken in order to evaluate what posture and breathing states contribute to best results in the sense that the scan volume and, therefore, the body density can be calculated. Initially a purely anthropometric approach was followed as well. For this, one of the postures was repeated twice (see above). That approach did not prove to be successful and was subsequently dropped.

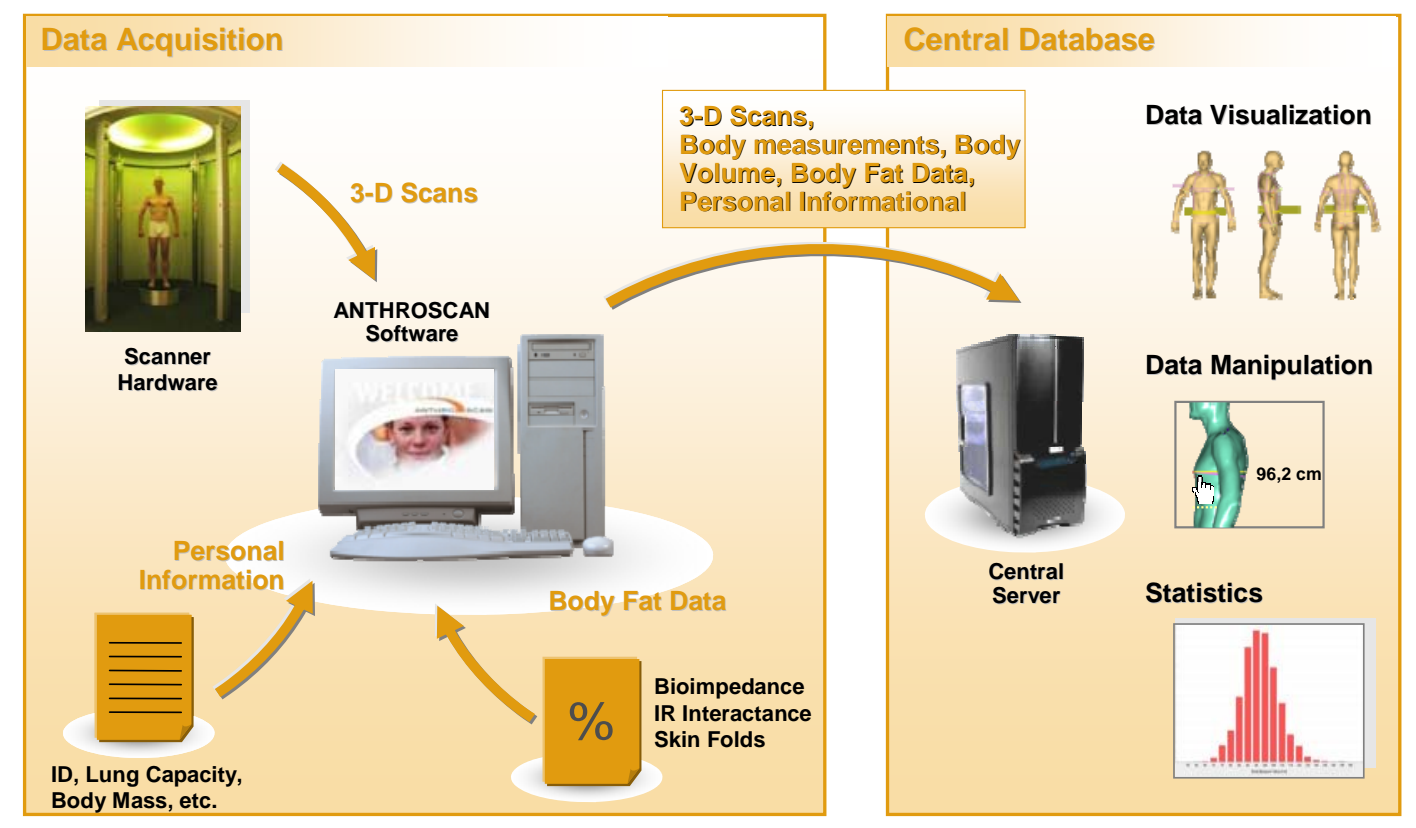

Figure 2: Infrastructure to collect and share data 
To determine a corrected net body volume considering lung air inclusions, vital capacity, expiratory reserve volume, and inspiratory reserve volume was collected as well.

Four skin fold thicknesses were collected for each subject at triceps, biceps, subscapular and suprailliac. In accordance with Durnin and Womersley those were transferred to body fat percentage considering the subjects age [3].

Each subject was given a unique ID and additional personal information such as gender, age, and body mass was taken. Additionally, a questionnaire was given to gain background information about each subject's physical activity and fitness level. This information was not considered in the course of the research described in this paper.

All data were collected with help of Human Solutions' ANTHROSCAN solution consisting of 3-D body scan hardware and software to process and collect addition information for each subject. A central database server to which all data were uploaded automatically gave access to all members of the project team (Figure 2).

\section{Test Data}

From the subjects' age it can be seen that the test population, with a mean age of 47,7 years for females and 49,6 years for males, is middle-aged rather than young or old. With respect to body type - although knowing that the body mass index is being discussed controversially - the test population seems to be slightly overweight with an mean BMI of 28,8 for females and 27,2 for males (Table 1 ). Also, females were overrepresented, with 102 subjects out of the total 141. I.e. data of 39 males were collected.

Table 1: Age distribution of test population

\begin{tabular}{|c|c|c|c|c|}
\hline Age [years] & Min & $\operatorname{Max}$ & Mean & Stand. Dev. \\
\hline Male & 25 & 65 & 47,7 & 10,8 \\
\hline Female & 23 & 75 & 49,6 & 11,0 \\
\hline $\mathrm{BMI}\left[\mathrm{kg} / \mathrm{m}^{2}\right]$ & Min & Max & Mean & Stand. Dev. \\
\hline Male & 20,4 & 43,2 & 28,8 & 5,7 \\
\hline Female & 19,0 & 42,8 & 27,2 & 5,1 \\
\hline
\end{tabular}

As explained above, the net body volume is needed to calculate body density and subsequently body fat percentage. For the net body volume air inclusions created by scan wear, hair, lung capacity, and the gastrointestinal tract must be subtracted from the gross body volume calculated from the 3-D scan. As it was not possible to measure subjects' net body volumes by means of hydrodensitometry, the body volumes were corrected empirically by taking into account the subjects' body measurements. The target values driving this optimization were the bioimpedance results.

It was found that accuracy increased using scans with a clearly defined breathing state-namely the posture with arms and legs splayed apart and completely exhaled (Figure 3). Therefore, all results were evaluated with data gained from that posture.
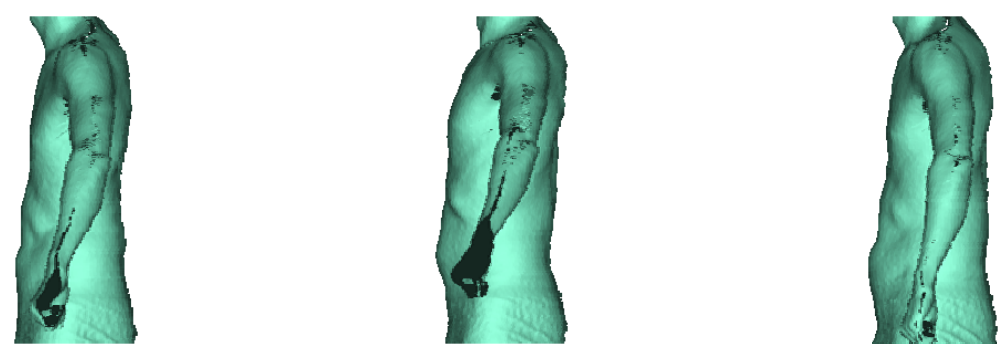

Figure 3: Torso shapes in various breathing shapes

In a further step, the body fat percentage in accordance to the approaches to Siri and Brozek were calculated using the corrected body net volume and body mass. A comparison of all body fat percentages with mean values and standard deviations is given in Table 2. 


\begin{tabular}{lcr} 
& Mean Value & Standard Deviation \\
\hline Skin Folds Method & $38,9 \%$ & $12,5 \%$ \\
Brozek, Scan & $26,0 \%$ & $11,6 \%$ \\
Siri, Scan & $27,8 \%$ & $9,3 \%$ \\
Bioimpedance & $27,8 \%$ & $8,9 \%$ \\
IR Interactance & $32,1 \%$ & $13,0 \%$
\end{tabular}

Table 2: Results body fat percentage

Means values of the body fat percentages varied broadly between $26 \%$ and $39 \%$ while standard deviations were very similar and ranged from $9 \%$ to $13 \%$.

Skin folds values deviate strongly from the values gained with bioimpedance, IR interactance and the new scan methods. Also other authors found that skin fold methods overestimate overall body fat percentage especially with overweight subjects $[1,8]$.

\begin{tabular}{lcc} 
& Deviation & Confidence Interval \\
\hline Brozek, Scan - Skin Folds & $+10 \% /-10 \%$ & $57,3 \%$ \\
Brozek, Scan - Bioimpedance & $+10 \% /-10 \%$ & $92,0 \%$ \\
Brozek, Scan - IR Interactance & $+10 \% /-10 \%$ & $86,7 \%$ \\
Siri, Scan - Skin Folds & $+10 \% /-10 \%$ & $59,3 \%$ \\
Siri, Scan - Bioimpedance & $+10 \% /-10 \%$ & $92,7 \%$ \\
Siri, Scan - IR Interactance & $+10 \% /-10 \%$ & $88,0 \%$ \\
IR Interactance - Bioimpedance & $+10 \% /-10 \%$ & $94,7 \%$ \\
IR Interactance - Skinfold & $+10 \% /-10 \%$ & $76,7 \%$ \\
Bioimpedance - Skinfold & $+10 \% /-10 \%$ & $60,7 \%$
\end{tabular}

Table 3: Comparison of methods with each other

Next, deviations for each two methods were determined and for $\pm 10 \%$ deviations confidence intervals were calculated (Table 3). As measurements of skin folds methods are farthest off from the other methods, confidence intervals are obviously lower, i.e. $77 \%$ and $61 \%$ for IR interactance and bioimpedance, and $57 \%$ and $59 \%$ for scanner-based methods.

As deviations between scanner-based and the non-skin folds methods at smaller confidence intervals are pleasantly high, ranging from $87 \%$ up to $93 \%$. Only IR interactance and bioimpedance show a better correlation of a confidence interval of $95 \%$.

\section{Results and Conclusion}

To determine overall body fat percentage a wide range of methods is available that can be broadly classified as direct methods, indirect methods, and physical measurement principles. Densitometry focuses on estimating body composition. Depending on information available, different component models can be used. For all component models, the body density is needed and is calculated from body mass and body volume. Therefore, it is feasible to employ 3-D whole body scanner systems to estimate whole-body fat percentage.

A survey was conducted in order to rank a 3-D body scanning approach into and compare it with existing methods. Data of 141 subjects were collected comprising of body values, 3-D whole body scans in various postures, body measurements, body fat percentages, and socio-demographic information - just to name a few.

A scanner-based approach was developed that is stable and practicable for work in common scannerapplication scenarios. Additionally it was found that the new scanner-based approach produces similar results to bioimpedance and is close to IR interactance measurements. Presumably due to the slightly overweight test sample, the new approach cannot be compared with skin folds measurements.

With this very promising outcome it is suggested to use the new approach in future set-ups in order to refine the method, learn more about the limitations, and to evaluate it further. 


\section{References}

1. Aghdassi, E., Arendt, B., Salit, I. E., Allard, J. P. (2007): "Estimation of Body Fat Mass Using Dual-Energy X-Ray Absorptiometry, Bioelectric Impedance Analysis, and Anthropometry in HIVPositive Male Subjects Receiving Highly Active Antiretroviral Therapy", Journal of Parenteral and Enteral Nutrition, No 31. pp. 135-141.

2. Brozek, J., Grande, F., Anderson, J. T., Keys, A. (1963): „Densitometric analysis of body composition: Revisions of some quantitiative assuptions", Annals of the new York Academy of Science, No. 110, pp. 113-140.

3. Durnin, J. G. A., Womersley, J. (1974): "Body fat assessed from total body density and its estimation from skinfold thickness: measurements on 481 men and women aged from 16 to 72 years. British Journal of Nutrition, No. 32, pp. 77-97.

4. Going, S. B. (2005): "Hydrodensitometry and Air Displacement Plethysmography", Human Body Composition, pp. 17-33.

5. Hoffman, D. J., Huber-Miller, R. K., Allison, D. B. , Wang, Z., Shen, W., Heymsfield, S. B. (2003); „Human Body Composition, published in "Obesity” edited by Lippincott, et al., pp. 103-146.

6. International Standard ISO 20685 (2005): "3-D scanning methodologies for internationally compatible anthropometric databases".

7. Siri, W. E. (1956): "The gross compositions of the body", Advances in biological and medical physics, No. 4, pp. 239-280.

8. Wattanapenpaiboon, N., Lukito, W., Strauss, B. J. G., Hsu-Hage, BH.-H., Wahlqvist, M. L., Stroud, D. B. (1998): „Agreement of skinfold measurement and bioelectrical impedance analysis methods with dual energy X-ray absorptiometry in estimating total body fat in Anglo-Celtic Australians“, International Journal of Obesity, Nr. 22, pp. 854-860. 